\title{
Serum Homocysteine, Lipid Profile and BMI as Atherosclerotic Risk Factors in Children with Numerical Chromosomal Aberrations
}

\section{Eman R. Youness}

National Research Centre

Sherien M El-Daly

National Research Centre

Hanaa Reyad Abdallah

National Research Centre

Hala T El-Bassyouni ( $\sim$ halabassyouni@yahoo.com )

National Research Centre https://orcid.org/0000-0001-9825-1219

Hisham Megahed

National Research Centre

Azzah A Khedr

National Research Centre

Marwa Elhady

Al-Azhar University

Walaa A Abuelhamid

Cairo University Kasr Alainy Faculty of Medicine

\section{Research Article}

Keywords: BMI, Down syndrome, homocysteinemia, Klinefelter, lipid profile, numerical chromosomal disorders, Turner syndrome

Posted Date: June 15th, 2021

DOl: https://doi.org/10.21203/rs.3.rs-595596/v1

License: (c) (i) This work is licensed under a Creative Commons Attribution 4.0 International License. Read Full License

Version of Record: A version of this preprint was published at World Journal of Pediatrics on April 16th, 2022. See the published version at https://doi.org/10.1007/s12519-022-00534-4. 


\section{Abstract}

Children with chromosomal disorders are at greater risk for cardiovascular comorbidities. Dyslipidemia and homocysteinemia have been identified to play a role in the pathogenesis of premature atherosclerosis. The current study aimed to explore the association of serum homocysteine level in children with numerical chromosomal disorders (Klinefelter syndrome, Down syndrome, and Turner syndrome) and explore its relation to dyslipidemia among such children. This case control study included 60 children with numerical chromosomal disorders (18 Klinefelter syndrome 22 Down syndrome and 20 Turner syndrome) diagnosed by cytogenetic analysis. In addition, 37 healthy normal weight children were included as control group. Anthropometric assessment was done for all groups including weight, height and body mass index was calculated. Serum level of homocysteine, cholesterol, triglycerides, high density lipoprotein and low-density lipoprotein were measured for all included children. Serum homocysteine level was significantly higher in children with numerical chromosomal disorders in comparison to healthy controls. Serum homocysteine level has significant positive correlation with cholesterol and low-density lipoprotein. Despite of the significant positive correlation between homocysteine serum levels and body mass index (BMI) in children with numerical chromosomal disorders, there was no significant difference in homocysteine level between children with and without obesity. Regression analysis demonstrated significant association between homocysteinemia and LDL level in children with chromosomal disorders (odd ratio: 1.396; 95\% confidence interval: 1.177-1.657). There is significant association between dyslipidemia and homocysteinemia in children with non-mosaic numerical chromosomal disorders that positively correlated with body mass index.

\section{Introduction}

Down syndrome, Turner syndrome, and Klinefelter syndrome are the most common chromosomal disorders worldwide. Down syndrome is caused by extra 21 chromosomes with incidence of 1 in 800 to 1000 live births that is easily identified at birth due to distinct features. Turner syndrome resulted from Xchromosome monosomy with incidence of 1 in 2000 of live-born female infants. While Klinefelter syndrome is caused by an extra $X$ chromosome in male infants with incidence of 1 in 600 live-born male infants. Diagnosis of Turner and Klinefelter syndromes is usually missed at birth; most of them are diagnosed in late childhood. In spite that each of them is caused by different changes in the chromosomes number, but all of them have greater liabilities for metabolic and cardiovascular comorbidities [1]. With advanced health care services, children with those chromosomal disorders have extended life span to adolescence and many of them reach adulthood. Complementary health care system is required for early detection of comorbidities among those children to improve their quality of life [2].

Several studies have demonstrated higher risk for cardiovascular disorders among children with chromosomal disorders including premature atherosclerosis and coronary heart disease that represent a major cause for mortality among those children [3-5]. Several non-traditional modifiable risk factors have been identified including homocysteinemia. Recognition of such risk factors is important for early 
identification and management of such comorbidities through establishing effective preventive measures [6].

Homocysteine is a sulfhydryl-containing amino acid produced as an intermediate product during the metabolism of methionine and cysteine. $70-80 \%$ of circulating homocysteine are bound to plasma proteins and $20-30 \%$ bound with itself or with other thiols, while $1 \%$ presented as free thiol.

Homocysteine is considered as a key element of the methylation cycle [7]. Homocysteinemia is caused either by genetic disorder which is rare but severe or secondary to other pathogenic disorders including chromosomal and environmental factors [8].

There is emerging evidence linking elevated circulating homocysteine level with dyslipidemia and cardiovascular morbidities including atherosclerosis, ischemic heart disease, thromboembolism, and cerebrovascular accident especially in those with other risk factors. Homocysteinemia is considered as an independent risk factor for cardiovascular mortality $[9,10]$. Elevated homocysteine induces vascular endothelium injury, smooth muscle cell proliferation and inflammation leading to the formation of atheromatous plaques, which can result in ischemic insults. Additionally, homocysteine decreases serum level of high-density lipoprotein (HDL) through suppressing apo-A lipoprotein synthesis and increase HDL clearance. Furthermore, homocysteine increases low-density lipoprotein (LDL), cholesterol peroxidation and thrombosis activation [11].

However, there is limited data regarding the relationship between circulating homocysteine level and dyslipidemia, especially in children with chromosomal disorders.

\section{Aim Of The Work:}

The current study aims to explore the relation between serum homocysteine level, lipid profile and Body Mass Index in children with non- mosaic numerical chromosomal disorders including Klinefelter syndrome, Down syndrome, and Turner syndrome for early detection of cardiovascular co-morbidities.

\section{Patients And Methods}

The current study is a cross sectional case control study included 60 children with documented diagnosis of non -mosaic numerical chromosomal disorders (aneuploidy); 18 children with Klinefelter syndrome (XXY), 22 cases as Down syndrome (trisomy 21) and 20 as Turner syndrome (monosomy X). In addition, 37 healthy normal children (body mass index between 5th and 85th percentiles) were included as control group. Children with chromosomal disorders were recruited consecutively from the Genetic clinic of both the Pediatric department, Faculty of Medicine, Cairo University, and the Clinical Genetic Department, National Research Centre, Egypt. While healthy controls were selected from Al-Zahraa hospital, Al-Azhar University, Cairo, Egypt. Both groups were matched for age. An informed written consent was received from parents of all included children in accordance with the guideline of ethics committee of Al-Azhar University. 


\section{Methods}

All the included children were subjected to detailed history taking with special emphasis in dietetic history, physical activity and lifestyle. Complete general and systematic examination was done. Anthropometric measurements including weight and height were done using standardized equipment. Body weight $(\mathrm{kg})$ was assessed while the child in light clothes, height $(\mathrm{cm})$ was measured while the child bare foot and knees stretched [12]. All parameters were recorded to the nearest 0.1 value. All measurements were plotted on age and sex specific growth charts [13].

Body mass index (BMI) was calculated as body weight in kilogram divided by square of height in meter. Obesity was defined as $\mathrm{BMI} \geq 95$ th percentiles while overweight was defined as $\mathrm{BMI} \geq 85$ th but less that 95th percentiles. Children were considered of normal BMI if their BMI ranged between the 5th and 85th percentiles for age and sex specific percentiles [14].

Children who have any acute medical illness, hepatic, or renal impairment or those who received drugs affecting lipid profile as corticosteroids were excluded.

\section{Laboratory investigations}

Peripheral blood samples were collected from both groups and subjected to conventional G- banding technique on blood lymphocytes [15].

\section{Lipid profile assessment}

Blood samples were collected after a 12-h overnight fasting and centrifuged to collect serum then stored at $<80$ _C until analyzed. An Olympus AU400 automatic analyzer (Olympus Corporation, Tokyo, Japan) was used to measure serum total cholesterol (TC), high density lipoprotein cholesterol (HDL-C), triglycerides (TG) and fasting blood glucose (FBG); fasting blood insulin (FBI) were measured with commercial kits (Roche Diagnostics, Indianapolis, IN, USA). While Low density lipoprotein-cholesterol (LDL-C) was calculated using Friedewald equation: LDL-C = TC - HDL-C- (TG/5).

\section{Quantification of Homocysteine}

Homocysteine serum level was determined using reversed phase High-performance liquid chromatography according to the method described by Melnyk et al., [16]. Homocysteine was separated using High-performance liquid chromatography, Agilent 1100 series with a reversed-phase $\mathrm{C} 18$ column (3 $\mu \mathrm{m}$ bead size; $3.9 \times 150 \mathrm{~mm}$ ). The mobile phase formed of $40 \mathrm{mmol} / \mathrm{L}$ sodium phosphate monobasic, monohydrate: $8 \mathrm{mmol} / \mathrm{L}$ heptane sulfonic acid and $18 \%(\mathrm{v} / \mathrm{v})$ methanol adjusted to $\mathrm{pH} 3.1$ which was filtered through a $0.45-\mu \mathrm{m}$ membrane filter. The isocratic elution had done by flow rate of $1.0 \mathrm{~mL} / \mathrm{min}$ at $40 \circ \mathrm{C}$. The detector wavelength was at $260 \mathrm{~nm}$.

\section{Statistical analyses}


Data analysis was performed using the statistical package for social sciences (SPSS, USA). Quantitative data was expressed as means \pm standard deviation. Differences between groups were analyzed using independent student $t$ test, ANOVA test and post hoc analysis. Pearson correlation coefficient test was used to assess correlations between investigated parameters, regression analysis was done also. Pvalues $<0.05$ were considered significant.

\section{Results}

The results are elucidated in Fig. (1). All patients were subjected to cytogenetic study of metaphase spreads. Chromosomes were arranged in Karyograms as shown in Figs. 2 (a, b, c). All samples had non mosaic aneuploidy in all cells as follows 18 children with Klinefelter syndrome (XXY), 22 cases as Down syndrome (trisomy 21) and 20 as Turner syndrome (monosomy X).

Serum homocysteine levels were significantly higher in children with chromosomal disorders in comparison to healthy controls. Furthermore, BMI, total cholesterol and LDL level were significantly higher in children with chromosomal disorders as shown in Tables 1 and 2. 
Table 1

Clinical data and lipid profile of the studied groups

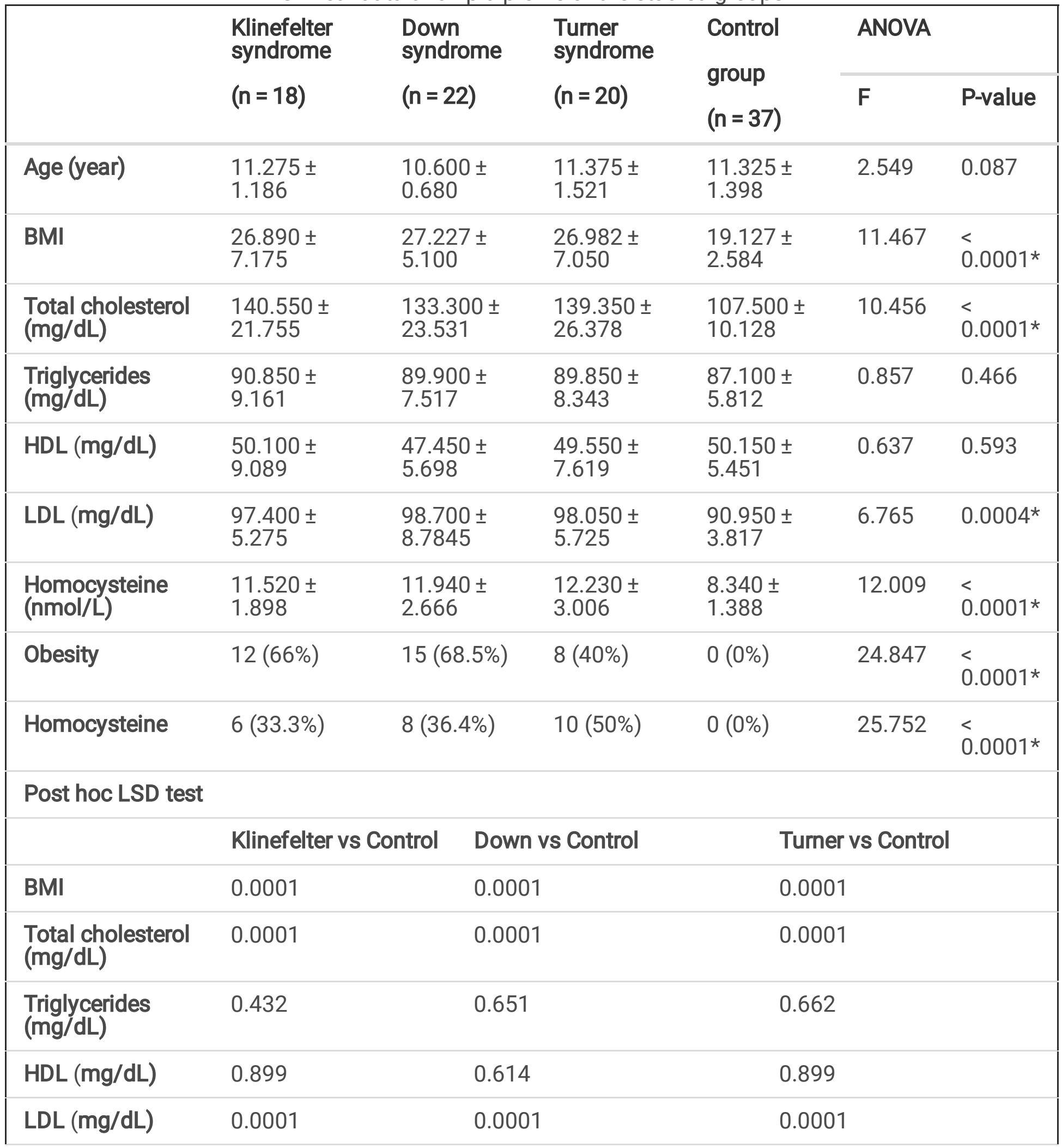

*Significant

BMI: body mass index; LDL: low density lipoprotein; HDL: high density lipoprotein; TC: total cholesterol 


\begin{tabular}{|c|c|c|c|c|c|c|}
\hline & $\begin{array}{l}\text { Klinefelter } \\
\text { syndrome }\end{array}$ & $\begin{array}{l}\text { Down } \\
\text { syndrome }\end{array}$ & $\begin{array}{l}\text { Turner } \\
\text { syndrome }\end{array}$ & Control & & \\
\hline & $(n=18)$ & $(n=22)$ & $(n=20)$ & $(n=37)$ & $F$ & P-value \\
\hline $\begin{array}{l}\text { Homocysteine } \\
\text { (nmol/L) }\end{array}$ & 0.0001 & 0.00 & & & & \\
\hline *Significant & & & & & & \\
\hline $\begin{array}{l}\text { BMI: body mass } \\
\text { cholesterol }\end{array}$ & LDL: lo & lipoprot & DL: high & lipop & & \\
\hline
\end{tabular}

Table 2

Correlation between lipid profile and homocysteine among the studied groups

\begin{tabular}{|c|c|c|c|c|c|c|c|}
\hline & & BMI & LDL & HDL & TG & TC & age \\
\hline \multirow[t]{2}{*}{ Klinefelter syndrome $(n=18)$} & $\mathbf{r}$ & 0.427 & 0.482 & 0.052 & -0.033 & 0.244 & -0.139 \\
\hline & $\begin{array}{l}\mathrm{p}- \\
\text { value }\end{array}$ & 0.077 & $0.042^{*}$ & 0.837 & 0.896 & 0.329 & 0.582 \\
\hline \multirow[t]{2}{*}{ Down syndrome $(n=22)$} & $\mathbf{r}$ & 0.685 & 0.696 & -0.022 & 0.090 & 0.486 & -0.174 \\
\hline & $\begin{array}{l}\mathrm{p}- \\
\text { value }\end{array}$ & $0.0004^{*}$ & $0.0003^{*}$ & 0.922 & 0.690 & $0.022 *$ & 0.438 \\
\hline \multirow[t]{2}{*}{ Turner syndrome $(n=20)$} & $\mathbf{r}$ & 0.569 & 0.665 & -0.231 & 0.303 & 0.443 & -0.396 \\
\hline & $\begin{array}{l}\text { p- } \\
\text { value }\end{array}$ & $0.005^{*}$ & $0.001^{*}$ & 0.327 & 0.194 & 0.050 & 0.083 \\
\hline \multirow{2}{*}{$\begin{array}{l}\text { Control group } \\
(n=37)\end{array}$} & $\mathbf{r}$ & -0.004 & -0.036 & 0.066 & 0.264 & -0.323 & -0.013 \\
\hline & $\begin{array}{l}\mathrm{p}- \\
\text { value }\end{array}$ & 0.981 & 0.832 & 0.697 & 0.114 & 0.051 & 0.939 \\
\hline \multirow{2}{*}{$\begin{array}{l}\text { All Chromosomal disorders } \\
(n=60)\end{array}$} & $\mathbf{r}$ & 0.654 & 0.667 & -0.094 & 0.214 & 0.389 & -0.217 \\
\hline & $\begin{array}{l}\text { p- } \\
\text { value }\end{array}$ & $0.0001 *$ & $0.0001 *$ & 0.407 & 0.056 & $0.002^{*}$ & 0.531 \\
\hline
\end{tabular}

*Significant

BMI: body mass index; LDL: low density lipoprotein; HDL: high density lipoprotein; TC: total cholesterol

Serum homocysteine level had significant positive correlation with low density lipoprotein in all groups of children with chromosomal disorders while homocysteine level had significant positive correlation with total cholesterol level only in children with Down syndrome. Homocysteine level had significant positive correlation with BMI in children with Down and Turner syndromes as demonstrated in Table 3. 
Table 3

Comparison between chromosomal disorders children with and without obesity as regarding homocysteine level

\begin{tabular}{|lllll|}
\hline & $\begin{array}{l}\text { chromosomal } \\
\text { disorders with obesity } \\
(\mathbf{n}=35)\end{array}$ & $\begin{array}{l}\text { chromosomal disorders } \\
\text { without obesity }(\mathbf{n = 2 5})\end{array}$ & \multicolumn{2}{l|}{$\begin{array}{l}\text { Independent } t \\
\text { test/chi square test }\end{array}$} \\
\cline { 4 - 6 } & & & $\mathrm{t} / \mathrm{X2}$ & P-value \\
\hline Homocysteine(nmol/L) & $12.948 \pm 2.087$ & $10.424 \pm 2.372$ & 4.247 & $<$ \\
\hline Homocysteinemia & $21(\% 60 \%)$ & $3(12 \%)$ & 12.0714 & $<$ \\
& & & & $0.0001^{*}$ \\
\hline
\end{tabular}

Despite of the significant positive correlation between homocysteine serum levels and body mass index in children with chromosomal disorders, there was no significant difference between homocysteine level in children with and without obesity as shown in Table 4.

Table 4

Logistic regression analysis for association of lipid profile level, BMI and homocysteinemia in children with chromosomal disorders

\begin{tabular}{|c|c|c|c|c|c|c|}
\hline \multicolumn{7}{|c|}{ Binary regression analysis } \\
\hline Variable & Coefficient & Std. Error & Wald & P-value & Odds ratio & $95 \% \mathrm{Cl}$ \\
\hline LDL (mg/dl) & 0.265 & 0.0976 & 7.392 & $0.006^{*}$ & 1.3041 & 1.076 to 1.579 \\
\hline BMI & 0.092 & 0.0698 & 1.759 & 0.184 & 1.0971 & 0.956 to 1.258 \\
\hline TC (mg/dl) & 0.004 & 0.017 & 0.083 & 0.773 & 1.0049 & 0.972 to 1.039 \\
\hline Constant & -29.876 & 9.064 & 10.862 & $0.001^{*}$ & - & - \\
\hline \multicolumn{7}{|c|}{ Stepwise regression analysis } \\
\hline Variable & Coefficient & Std. Error & Wald & P-value & Odds ratio & $95 \% \mathrm{Cl}$ \\
\hline LDL (mg/dl) & 0.3341 & 0.087 & 14.670 & $0.0001 *$ & 1.3967 & 1.177 to 1.657 \\
\hline Constant & -33.337 & 8.642 & 14.880 & $0.0001^{*}$ & - & - \\
\hline *significant & & & & & & \\
\hline
\end{tabular}

Binary and stepwise logistic regression analysis demonstrated significant association between homocysteinemia and LDL level in children with chromosomal disorders as shown in Table 5.

\section{Discussion}


Subjects with chromosomal disorders are at higher risk for cardiometabolic comorbidities that develop earlier than in the general population. Homocysteinemia has been emerged as an independent risk factor for cardiovascular diseases. Reports showed that about $40 \%$ of patients with cerebrovascular disorders have homocysteinemia. There are controversial reports regarding the relationship between homocysteine and lipid profile under several normal and pathological conditions 16 . However, there is no sufficient data exploring the prevalence of homocysteinemia in children with chromosomal disorders and its relation to dyslipidemia.

There is controversy in the identification of the cutoff level of homocysteinemia in children and adolescents that varied according to age and ethnicity between 8.3 and $13.75 \mathrm{nmol} / \mathrm{L}$. In the current study we relied on a cutoff level $>95$ th percentile for age and sex to identify homocysteinemia $[17,18]$.

The current study demonstrated higher homocysteine serum level in children with Klinefelter, Turner and Down syndromes in comparison to healthy children with positive correlation between homocysteine level and BMI. These findings could be partially explained by higher rate of overweight and obesity among children with chromosomal disorders especially those with Down syndrome. These findings are in accordance with Kumar et al. [19], who reported that among school aged children none of normal weight children had homocysteinemia while $37.5 \%$ of overweight children and $36.5 \%$ of obese children had homocysteinemia. However, the frequencies of homocysteinemia in obese children with chromosomal disorders much exceed that observed in healthy children.

However, further analysis of our results demonstrated that homocysteine level was significantly higher in normal weight children with chromosomal disorders when compared to normal weight healthy children suggesting the presence of other causes for homocysteinemia rather than increased BMI. This indicates that numerical chromosomal aberrations play a role in the development of homocysteinemia.

Schulze et al. [20] suggested that homocysteinemia in children with numerical chromosomal aberrations could be due to genetic polymorphisms, physical activity patterns, adiposity, and nutritional deficiency of folic acid and vitamin B12. In children with Turner syndrome, previous reports demonstrated that homocysteinemia may be related to female sex hormones deficiency. Estrogen deficiency caused by monosomy $\mathrm{X}$ chromosome may represent a possible cause for homocysteinemia in children with Turner syndrome [21]. While homocysteinemia in children with Klinefelter syndrome may be related to increased muscle mass. Furthermore, previous evidence showed increased homocysteine level after treatment with testosterone in patients with Klinefelter syndrome [22].

Our results demonstrated higher levels of total cholesterol and LDL in children with homocysteinemia. Regression analysis revealed a significant association between homocysteinemia and LDL level. These findings are in accordance with previous studies that have shown strong association between homocysteinemia and dyslipidemia. This could be attributed to the effect of homocysteine that promotes the formation and secretion of cholesterol by the hepatocytes that may contribute to the direct correlation between cholesterol and homocysteine level [23]. Experimental studies demonstrated that homocysteine 
thiolactone triggers the aggregation of LDL leading to the formation of foam cells in cultured human macrophages [24].

Several studies demonstrated the role of homocysteine in enhancement of low-density lipoprotein peroxidation. Dong et al., [25] reported significant positive correlation between homocysteine and LDL level in patients with hypothyroidism, which is commonly associated with both Down syndrome and Turner syndrome. Furthermore, reduction of homocysteine level was associated with decreased LDL.

Our findings came in agreement with Yakub et al. [26] who reported that homocysteinemia directly correlated with hypertension, dyslipidemia, and obesity. Experimental studies have demonstrated that hypomethylation due to homocysteinemia may attribute to visceral fat accumulation. Furthermore, homocysteinemia may also alter the activity of some inhibitory enzymes which are involved in HDL metabolism [27].

On the other hand, previous studies reported an association between homocysteinemia and the metabolic syndrome. Additionally, it was reported that homocysteinemia has a thrombogenic and atherogenic effect that attribute to premature atherosclerosis leading to several cardiometabolic events including stroke and ischemic heart disease [28].

In conclusion, our findings suggest a strong association between homocysteinemia and dyslipidemia in children with non- mosaic numerical chromosomal disorders who are known to have high risk of cardiovascular morbidities. Furthermore, BMI positively correlated with homocysteinemia in children with numerical chromosomal aberrations but not in children with normal karyotypes. Thus, numerical chromosomal aberrations may play a role in increased BMI.

Further studies are required to understand the causes of homocysteinemia in such children. Moreover, longitudinal follow up studies are needed to prove a cause-effect relationship between homocysteinemia and dyslipidemia in children with non- mosaic numerical chromosomal aberrations and assess cardiovascular comorbidities related to homocysteinemia.

\section{Limitations Of The Study}

There is no available cutoff point for homocysteine level for Egyptian children, however we used a cutoff point $>95$ th percentile that better reflects the risk of homocysteinemia in the Mediterranean populations.

\section{Declarations}

\section{Conflicts of interest}

No conflict of interests.

\section{Acknowledgments}


Authors thanks Faculty of Medicine \& El- Azhar universities and National Research Centre, Egypt for their help.

\section{References}

1. Tyler, C. and Edman, JC. (2004) Down syndrome, Turner syndrome, and Klinefelter syndrome: primary care throughout the life span. Prim Care. 31, 627-48.

2. Cohen, JS. and Biesecker, BB. (2010) Quality of life in rare genetic conditions: a systematic review of the literature. Am J Med Genet A. 152A, 1136-56.

3. Sobey, CG., Judkins, CP., Sundararajan, V., Phan, TG., Drummond, GR., Srikanth, VK. (2015) Risk of Major Cardiovascular Events in People with Down Syndrome. PLoS One. 10, e0137093.

4. Pasquali, D., Arcopinto, M., Renzullo, A., Rotondi, M., Accardo, G., Salzano, A., et al. (2013) Cardiovascular abnormalities in Klinefelter syndrome. Int J Cardiol.168, 754-9.

5. Kim, HK., Gottliebson, W., Hor, K., Backeljauw, P., Gutmark-Little, I., Salisbury, SR., et al. (2011) Cardiovascular anomalies in Turner syndrome: spectrum, prevalence, and cardiac MRI findings in a pediatric and young adult population. AJR Am J Roentgenol.196, 454-60.

6. Han L, Wu Q, Wang C, Hao Y, Zhao J, Zhang L, et al.(2015) Homocysteine, Ischemic Stroke, and Coronary Heart Disease in Hypertensive Patients: A Population-Based, Prospective Cohort Study. Stroke. 46, 1777-86.

7. Ganguly, P., Alam, SF. (2015) Role of homocysteine in the development of cardiovascular disease. Nutr J. 14:6.

8. Gravina-Taddei, CF., Batlouni, M., Sarteschi, C., Baltar, VT., Salvarini, NA., Bertolami, MC., et al. (2005) Hyperhomocysteinemia as a risk factor for coronary atherosclerotic diseases in the elderly. Arq Bras Cardiol. 85, 166-73.

9. Xiao, Y., Zhang, Y., Lv, X., et al. (2011) Relationship between lipid profiles and plasma total homocysteine, cysteine and the risk of coronary artery disease in coronary angiographic subjects. Lipids Health Dis. 10:137.

10. Napal Lecumberri, JJ., González Bores, P., Cuesta Marín, A., Caballero Avendaño, FA., Olmos Martínez, JM., Hernández Hernández, JL. (2020) Lipid profile and serum folate, vitamin B12 and homocysteine levels in patients with retinal vein occlusion. Clin Investig Arterioscler. S02149168(20)30085-1.

11. Liao, D., Tan, H., Hui, R., Li, Z., Jiang, X., Gaubatz, J., et al. (2006) Hyperhomocysteinemia decreases circulating high-density lipoprotein by inhibiting apolipoprotein A-I Protein synthesis and enhancing HDL cholesterol clearance. Circ Res. 199, 598-606.

12. Ayele, B., Aemere, A., Gebre, T., et al. (2012) Reliability of measurements performed by communitydrawn anthropometrists from rural Ethiopia. PLoS One. 7: e30345.

13. Ghalli, I., Salah, N., Hussien, F., Erfan, M., El-Ruby ,M., et al. (2008) In: Proceedings of the 1st National Congress for Egyptian Growth Curves, Cairo University, 11 December 2003. Published in: Sartorio A, 
Buckler JM, Marazzi N. Egyptian Growth Curves 2002 for Infants, Children and Adolescents. Crescere nel mondo, Ferring Publisher.

14. WHO. (2014) Global status report on noncommunicable diseases. Chapter 7, 79-94.

15. Verma, RS., Babu, A. (1995) Human Chromosomes Principles and Techniques 2nd edn. New York, McGraw- Hill.

16. Melnyk, S., Pogribna, M., Pogribny, IP., Yi, P. and James SJ. (2000) Measurement of plasma and intracellular S-adenosylmethionine and S-adenosylhomocysteine utilizing coulometric electrochemical detection: alterations with plasma homocysteine and pyridoxal 5'-phosphate concentrations. Clin Chem. 46, 265-272.

17. Momin, M., Jia, J., Fan, F., et al. (2017) Relationship between plasma homocysteine level and lipid profiles in a community-based Chinese population. Lipids Health Dis.16, 54.

18. Papandreou, D., Mavromichalis, I., Makedou, A., Rousso, I., Arvanitidou, M. (2006) Reference range of total serum homocysteine level and dietary indexes in healthy Greek schoolchildren aged 6-15 years. Br J Nutr. 96, 719-24.

19. Kumar, KJ., Saldanha, K., Sushma, K., Murthy, DS., Vishwanath, PA. (2017) Prospective Study of Homocysteine and its relation to Body Mass Index and Lipid Profile in School Children. Indian Pediatr. 54, 935-937.

20. Schulze, K.J., Yakub, M., Christian, P., Stewart, C.P. West, K.P. (2013) Prevalence and Determinants of Hyperhomocysteinemia in School Aged Children in Rural Nepal. FASEB J. 27, 1055.24.

21. Calcaterra, V., Larizza, D., De Giuseppe, R., et al. (2019) Diet and Lifestyle Role in Homocysteine Metabolism in Turner Syndrome. Med Princ Pract. 1, 48-55.

22. Yesilova, Z., Ozata, M., Oktenli, C., Sanisoglu, SY., Erbil, MK., Dagalp, K. (2004) Effect of supraphysiologic doses of testosterone on fasting plasma total homocysteine concentrations in men with Klinefelter's syndrome. Fertil Steril. 81, 1278-82.

23. Karmin, O., Lyme, EG., Chung, YH., et al. (1998) Homocysteine stimulates the production and secretion of cholesterol in hepatic cells. Biochem Biophys Acta 1323, 317-324.

24. Naruszewicz, M., Mirkiewicz, E., Olszewski, AJ., et al. (1994) Thiolation of low-density lipoprotein by homocysteine thiolactone causes increased aggregation and altered interaction with cultured macrophages. Nutr Metab Cardiovas Dis 4, 70-77.

25. Dong, X., Yao, Z., Hu, Y., et al. (2016) Potential harmful correlation between homocysteine and lowdensity lipoprotein cholesterol in patients with hypothyroidism. Medicine (Baltimore). 95, e4291.

26. Yakub, M., Schulze, KJ., Khatry, SK., Stewart, CP., Christian, P., West, KP. (2014) High plasma homocysteine increases risk of metabolic syndrome in 6- to 8-year-old children in rural Nepal. Nutrients. 6, 1649-61.

27. Obeid, R., Herrmann, W. (2009) Homocysteine and lipids: S-adenosyl methionine as a key intermediate. FEBS Lett. 583, 1215-1225. 
28. Guilland, JC., Favier, A., Potier de Courcy, G., Galan, P., Hercberg, S. (2003) Hyperhomocysteinemia: An independent risk factor or a simple marker of vascular disease? I. Basic data. Pathol. Biol. 51, 101110.

\section{Figures}

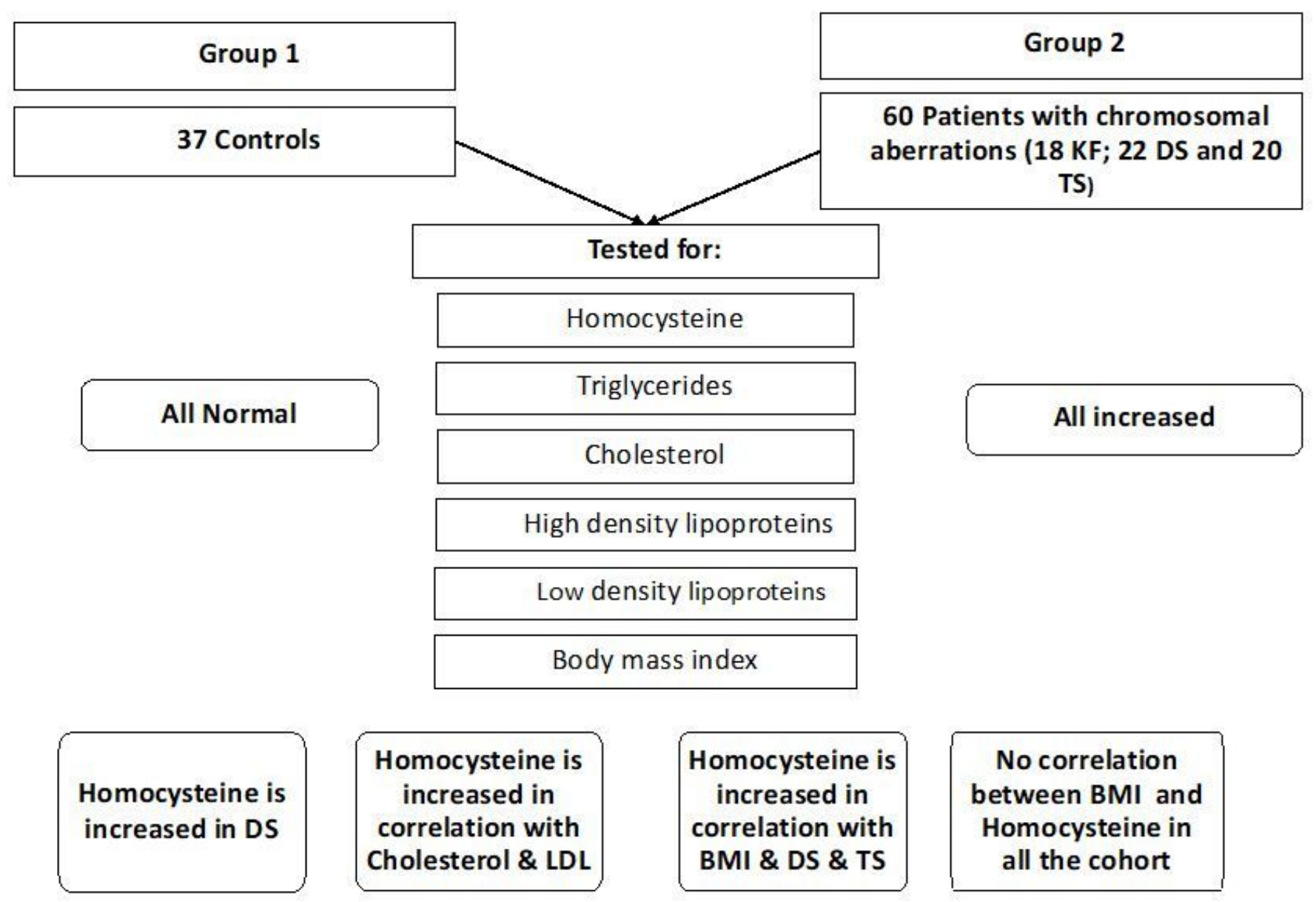

Figure 1

Algorithm elucidating our results 


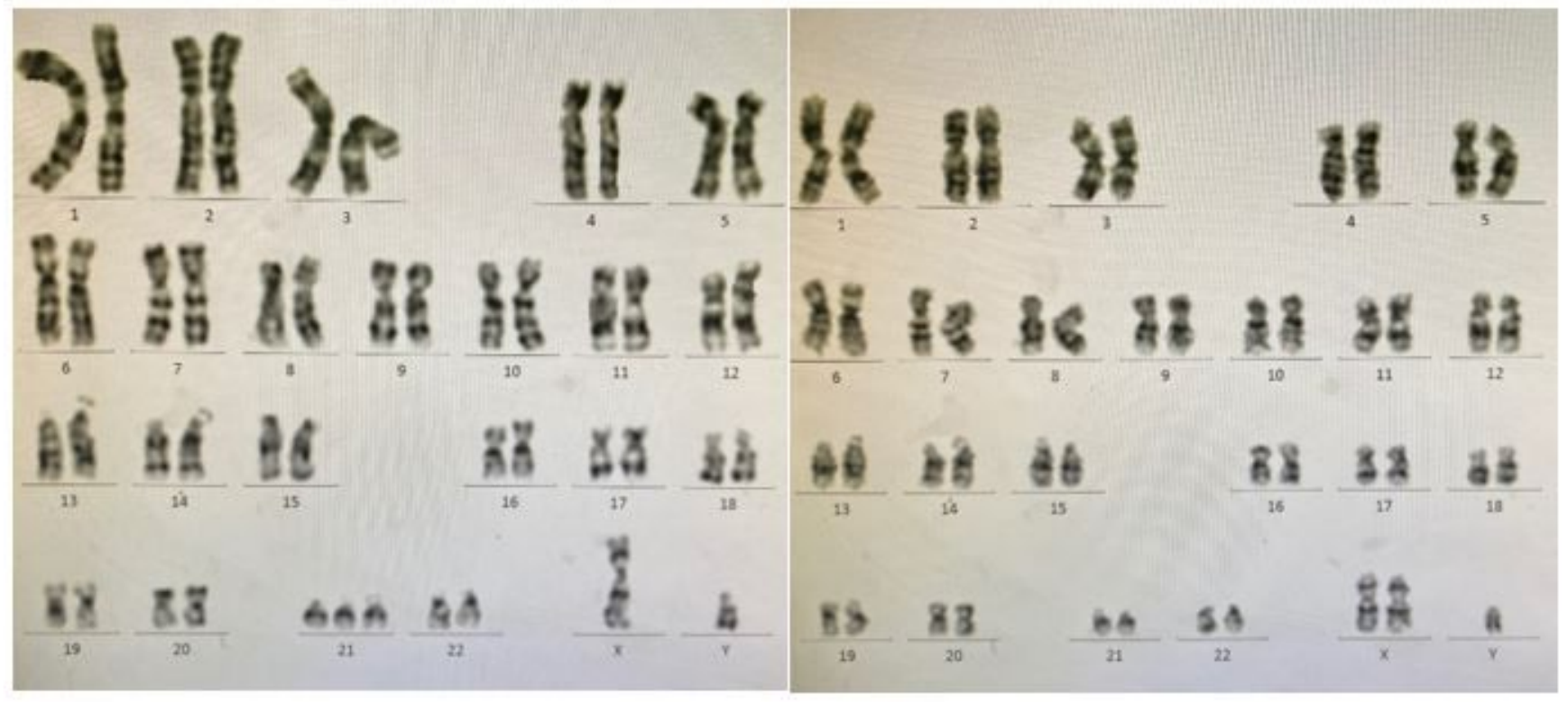

(a)

(b)

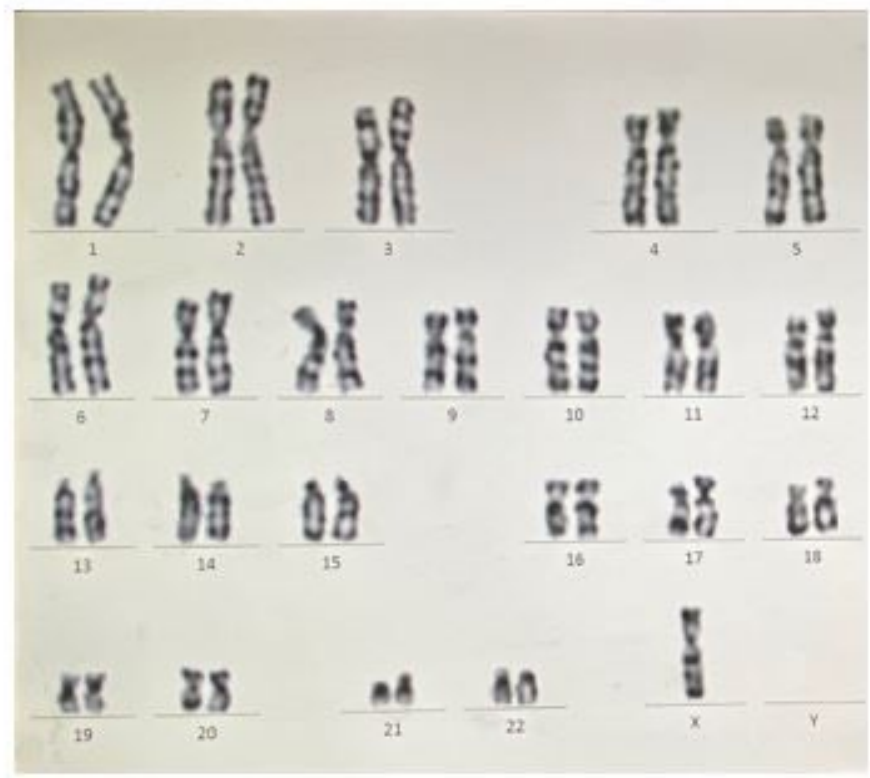

(c)

\section{Figure 2}

Karyograms for patients with: (a) Down syndrome with trisomy 21, (b) Klinefelter syndrome with XXY and (c) Turner Syndrome with loss of one X chromosome. 